\title{
On a new extension of the zero-divisor graph (II)
}

\author{
A. Cherrabi, H. Essannouni, E. Jabbouri, , A. Ouadfel* \\ Laboratory of Mathematics, Computing and \\ Applications-Information Security (LabMia-SI) \\ Faculty of Sciences, Mohammed-V University in Rabat. \\ Rabat. Morocco.
}

\begin{abstract}
We continue our study of the new extension of zero-divisor graph introduced in [5]. We give a complete characterization for the possible diameters of $\widetilde{\Gamma}(R)$ and $\widetilde{\Gamma}\left(R\left[x_{1}, \ldots, x_{n}\right]\right)$, we investigate the relation between the zero-divisor graph, the subgraph of total graph on $Z(R)^{\star}$ and $\widetilde{\Gamma}(R)$ and we present some other properties of $\widetilde{\Gamma}(R)$.
\end{abstract}

\section{Introduction}

The zero-divisor graph of a commutative ring $R$ with $1 \neq 0$ was first introduced by Beck [4, where he was interested in colorings. In his work all elements of the ring were vertices of the graph and two distinct elements $x$ and $y$ are adjacent if and only if $x y=0$. D.F. Anderson and P.S. Livingston have defined a graph, $\Gamma(R)$, with vertices in $Z(R)^{\star}=Z(R) \backslash\{0\}$, where $Z(R)$ is the set of non-zero zero-divisors of $R$, and for distinct $x, y \in Z(R)^{\star}$, the vertices $\mathrm{x}$ and $\mathrm{y}$ are adjacent if and only if $x y=0[3]$. Also, D. F Anderson and A. Badawi introduced the total graph $T(\Gamma(R))$ of a commutative ring $R$ with all elements of $R$ as vertices and for distinct $x, y \in R$, the vertices $x$ and $y$ are adjacent if and only if $x+y \in Z(R)[2] Z^{\star}(\Gamma(R))$ denotes the induced subgraph of $T(\Gamma(R))$ where the vertices are the nonzero zero-divisor of $R$.

In [5], we introduced a new graph, denoted $\widetilde{\Gamma}(R)$, as the undirected simple graph whose vertices are the nonzero zero-divisors of $R$ and for distinct

\footnotetext{
${ }^{*}$ Corresponding Author: aliouadfel@gmail.com
} 
$x, y \in Z(R)^{\star}, x$ and $y$ are adjacent if and only if $x y=0$ or $x+y \in Z(R)$. Recall that a simple graph $G=(V, E)$ is connected if there exists a path between any two distinct vertices. A graph of order 0 or 1 is called trivial. For distinct vertices $x$ and $y$ of $G$, the distance $d(x, y)$ is the length of the shortest path connecting $x$ and $y$; if there is no such path, $d(x, y)=\infty$. The diameter of $G$ is $\operatorname{diam}(G)=\sup \{d(x, y) / x, y \in V$ and $x \neq y\}$. $G$ is complete if it is connected with diameter one and $K_{n}$ denote the complete graph with $n$ vertices. A hamiltonian cycle of $G$ is a spanning cycle of $G$. Also, $G$ is said to be hamiltonian if $G$ has a hamiltonian cycle. Basic reference for graph theory is [6].

As usual, $T(R)$ denotes the total ring of fractions of $R, N i l(R)$ the nilradical of $R$. General reference for commutative ring theory is [1].

In this paper, we continue our study of the graph $\widetilde{\Gamma}(R)$. In the first section, we completely characterize, in the general case, when $\widetilde{\Gamma}(R)$ and $\widetilde{\Gamma}\left(R\left[x_{1}, \ldots, x_{n}\right]\right)$ are complete graphs. In section 2 , We extend our study of cases where $\widetilde{\Gamma}(R)=\Gamma(R)$ and $\widetilde{\Gamma}(R)=Z^{\star}(\Gamma(R))$ started in [5] to the general case. The section 3 is devoted to giving some other properties of the graph $\widetilde{\Gamma}(R)$.

\section{When $\widetilde{\Gamma}(R)$ is complete}

In this section, we provide sufficient and necessary conditions for $\widetilde{\Gamma}(R)$ to be complete. This result is a generalization of our result concerning the case where $R$ is finite (cf. theorem 2.4 [5]). We recall that $R \simeq R_{1} \times \cdots \times R_{n}$, where $R_{1}, \ldots, R_{n}$ are non-trivial rings, if and only if there exists $e_{1}, \ldots, e_{n} \in$ $R^{\star}$ such that $\forall i, e_{i}$ is idempotent, $\sum_{i=1}^{n} e_{i}=1$ and if $i \neq j, e_{i} e_{j}=0$ (cf. proposition 2.1.1 [7]). Also, it is clear that $Z(T(R))=\left\{\frac{x}{s} / x \in Z(R), s \in\right.$

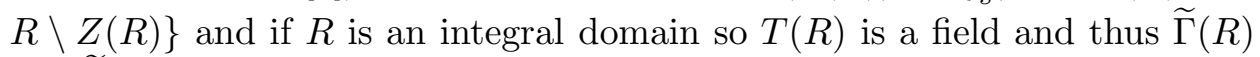
and $\widetilde{\Gamma}(T(R))$ are trivial. We agree that a trivial graph is complete.

Proposition 1.1. $\widetilde{\Gamma}(R)$ is complete if and only if $\widetilde{\Gamma}(T(R))$ is complete.

Proof. Suppose that $\widetilde{\Gamma}(R)$ is not trivial.

$\Rightarrow)$ : Let $\frac{x}{s}, \frac{y}{t} \in Z(T(R))^{\star}$ such that $\frac{x}{s} \neq \frac{y}{t}$ so $x, y \in Z(R)^{\star}$ hence $t x, s y \in$ $Z(R)^{\star}$ and $t x \neq s y$ therefore $t x$ and $s y$ are adjacent in $\widetilde{\Gamma}(R)$ because $\widetilde{\Gamma}(R)$ is complete. If tx.sy $=0$ so $x y=0$ hence $\frac{x}{s} \cdot \frac{y}{t}=0$. If $t x+s y \in Z(R)$ so $\frac{x}{s}+\frac{y}{t}=\frac{t x+s y}{t s} \in Z(T(R))$.

$\Leftarrow)$ : Let $x, y \in Z(R)^{\star}$ such that $x \neq y$ then $\frac{x}{1}, \frac{y}{1} \in Z(T(R))^{\star}$ and $\frac{x}{1} \neq \frac{y}{1}$ so $\frac{x}{1}$ and $\frac{y}{1}$ are adjacent in $\widetilde{\Gamma}(T(R))$. If $\frac{x}{1} \frac{y}{1}=0$ so $x y=0$ and if $\frac{x}{1}+\frac{y}{1} \in Z(T(R)$ so $x+y \in Z(R)$. 
Remark 1.2. Since diameters of $\widetilde{\Gamma}(R)$ and $\widetilde{\Gamma}(T(R)$ ) are at most 2 (cf. theorem 2.1 [5]), then $\operatorname{diam}(\widetilde{\Gamma}(T(R)))=\operatorname{diam}(\widetilde{\Gamma}(R))$.

Below, we give some useful lemmas to prove our first main result.

Lemma 1.3. Let $R \simeq R_{1} \times \cdots \times R_{n}$, where $n \geq 3$ and $R_{1}, \ldots, R_{n}$ are nontrivial rings. If $\widetilde{\Gamma}(R)$ is complete then $R$ is boolean.

Proof. Let $x_{1} \in R_{1}$ so $a=\left(x_{1}, 1,0, \ldots, 0\right) \in Z(R)^{\star}$, also $b=\left(1-x_{1}, 0,1, \ldots, 1\right) \in$ $Z(R)^{\star}$; we have $a \neq b$ and $a+b=(1, \ldots, 1) \notin Z(R)$ so $0=a b$ because $\widetilde{\Gamma}(R)$ is complete hence $x_{1}^{2}=x_{1}$. In the same way, $R_{2}, \ldots, R_{n}$ are boolean and thus $R$ is boolean.

Lemma 1.4. If $R$ is (up to isomorphism) a subring of a product of two integral domains, then $\widetilde{\Gamma}(R)$ is complete.

Proof. We can suppose that $R$ is a subring of $R_{1} \times R_{2}$, where $R_{1}, R_{2}$ are integral domains, and that $\widetilde{\Gamma}(R)$ is not trivial. Then there exists $a, b \in Z(R)^{\star}$ such that $a b=0$. Since $Z(R)$ is a subset of $Z\left(R_{1} \times R_{2}\right)=\left(R_{1} \times\{0\}\right) \cup(\{0\} \times$ $\left.R_{2}\right)$, we can suppose that $a \in R_{1} \times\{0\}$ and $b \in\{0\} \times R_{2}$. Let $x, y \in Z(R)^{\star}$ such that $x \neq y$. If $x, y \in R_{1} \times\{0\}$ (resp. $x, y \in\{0\} \times R_{2}$ ) then $(x+y) b=0$ (resp. $(x+y) a=0$ ) therefore $x+y \in Z(R)$. If $x \in R_{1} \times\{0\}$ and $y \in\{0\} \times R_{2}$ (resp. $x \in\{0\} \times R_{2}$ and $y \in R_{1} \times\{0\}$ ) then $x y=0$.

Definition 1.5. [7] $R$ is said to be indecomposable if $R$ cannot be decomposed into a direct product of two nonzero rings. Otherwise, $R$ is said to be decomposable.

Lemma 1.6. (lemma 2.4.9,[7]) $R$ is an indecomposable ring if and only if it has no nontrivial idempotents.

Theorem 1.7. $\widetilde{\Gamma}(R)$ is complete if and only if $R$ is boolean or $Z(R)$ is an ideal of $R$ or $R$ is (up to isomorphism) a subring of a product of two integral domains.

Proof. $\Leftarrow$ ): If $R$ is boolean then $\widetilde{\Gamma}(R)$ is complete (cf. the proof of the theorem 2.4 [5]). Also, it is obvious that if $Z(R)$ is an ideal of $R$ then $\widetilde{\Gamma}(R)$ is complete. If $R$ is a subring of a product of two integral domains, it follows from the lemma 1.4 that $\widetilde{\Gamma}(R)$ is complete.

$\Rightarrow)$ : Suppose that $Z(R)$ is not an ideal of $R$ so there exists $a, b \in Z(R)^{\star}$ such that $a+b \notin Z(R)$ so $a \neq b$ and since $\widetilde{\Gamma}(R)$ is complete, $a b=0$. Let $s=a+b, e=\frac{a}{s}$ so $e \in Z(T(R))^{\star}, 1-e=\frac{b}{s}$ and $e(1-e)=0$ because $a b=0$ then $e$ is a nontrivial idempotent in $T(R)$ and thus $T(R)=A_{1} \times A_{2}$, where 
$A_{1}, A_{2}$ are nontrivial rings.

We claim that if $A_{1}$ or $A_{2}$ contains a nontrivial idempotent, then $R$ is boolean, indeed, if there exists a nontrivial idempotent in $A_{1}$ (the other case is similar) then $A_{1}$ is a product of two rings. Since $\widetilde{\Gamma}(R)$ is complete, it follows from the proposition 1.1 that $\widetilde{\Gamma}(T(R))$ is complete thus according to the lemma 1.3, $T(R)$ is boolean and thus $R$ is boolean.

We claim that if the only idempotents of $A_{1}$ and $A_{2}$ are 0 and 1 then $A_{1}$ and $A_{2}$ are integral domains, indeed, let $x_{1} \in Z\left(A_{1}\right)$, we consider $x=\left(x_{1}, 1\right), y=\left(1-x_{1}, 0\right)$, we have $x, y \in Z(T(R))^{\star}$ and $x \neq y$. Since $\widetilde{\Gamma}(T(R))$ is complete and $x+y=(1,1) \notin Z(T(R)), x y=0$ so $x_{1}^{2}=x_{1}$ hence $x_{1}=0$. In the same way, we show that $A_{2}$ is an integral domain and thus $R$ is a subring of a product of two integral domains.

In the rest of this section, we will be interested in the case where the ring is a ring of polynomials. Let's start by recalling McCoy's theorem.

Theorem 1.8 (McCoy's Theorem, [8]). a polynomial $p(x) \in R[x]$ is a zerodivisor in $R[x]$ if and only if there is a nonzero element $r$ of $R$ such that $r p(x)=0$.

We recall that a ring $R$ satisfies property $\mathrm{A}$ if each finitely generated ideal $I \subset Z(R)$ has nonzero annihilator (cf. [8], p. 4). As a first step we prove the following lemma.

Lemma 1.9. the following statements are equivalent:

(a) $Z(R[x])$ is an ideal of $R[x]$.

(b) $R$ satisfies property $A$ and $Z(R)$ is an ideal of $R$.

(c) If $I$ is an ideal of $R$ generated by a finite number of zero-divisors, then $\operatorname{ann}_{R}(I) \neq(0)$.

Proof. According to theorem 3.3 [9], (a) and (b) are equivalent. (a) $\Rightarrow$ $(c))$ : Suppose that $Z(R[x])$ is an ideal of $R[x]$. Let $I=\left(a_{1}, \ldots, a_{n}\right)$, where $a_{1}, \ldots, a_{n} \in Z(R)^{\star}$, then $a_{1} X+\cdots+a_{n} X^{n} \in Z(R[X])$ so, according to McCoy's theorem, there exist $b \in Z(R)^{\star}$ such that $b\left(a_{1} X+\cdots+a_{n} X^{n}\right)=0$ therefore $\forall i=1, \ldots, n, b a_{i}=0$ hence $\operatorname{ann}(I) \neq(0)$.

$(c) \Rightarrow(b))$ : Suppose that for every ideal $I$ of $R$ generated by a finite number of zero-divisors, $\operatorname{ann}_{R}(I) \neq(0)$. It is obvious that $R$ satisfies property A. Let $a, b \in Z(R)^{\star}$, we consider $I=(a, b)$ then there exists $c \in R^{\star}$ such that $c a=c b=0$ so $c(a+b)=0$ hence $a+b \in Z(R)$. 
Theorem 1.10. $\widetilde{\Gamma}(R[x])$ is complete if and only if $R$ is (up to isomorphism) a subring of a product of two integral domains or $R$ satisfies one of the equivalent statements of lemma 1.9.

Proof. $\Rightarrow)$ : Since $x^{2} \neq x, R[x]$ is not boolean so, according to the theorem 1.7, $R[x]$ is a subring of a product of two integral domains or $Z(R[x])$ is an ideal of $R[x]$. If $R[x]$ is a subring of a product of two integral domains, then $R$ is too.

$\Leftarrow$ ): If $R$ is a subring of $R_{1} \times R_{2}$, where $R_{1}, R_{2}$ are integral domains, then it is obvious that $R[x]$ is a subring of $R_{1}[x] \times R_{2}[x]$. Since $R_{1}[x], R_{2}[x]$ are integral domains then, according to theorem 1.7, $\widetilde{\Gamma}(R[x])$ is complete. If $Z(R[X])$ is an ideal of $R[x]$ so $\widetilde{\Gamma}(R[x])$ is complete.

Lemma 1.11. $\widetilde{\Gamma}(R[x, y])$ is complete if and only if $\widetilde{\Gamma}(R[x])$ is complete.

Proof. $\Rightarrow)$ : is an immediate consequence of theorem 1.10

$\Leftarrow)$ : Since $R[x]$ is not boolean so, according to theorem $1.7, R[x]$ is isomorph to a subring of a product of two integral domains or $Z(R[x])$ is an ideal of $R[x]$. Since $R[x]$ satisfied property $\mathrm{A}$, then, according to theorem 1.10 , $\widetilde{\Gamma}(R[x, y])$ is complete.

Thus, For a ring of polynomials in $n$ indeterminates, we obtain:

Theorem 1.12. $\widetilde{\Gamma}\left(R\left[x_{1}, \ldots, x_{n}\right]\right)$ is complete if and only if $R$ is (up to isomorphism) a subring of a product of two integral domains or $R$ satisfies one of the equivalent statements of lemma 1.9.

Proof. According to lemma 1.11, $\widetilde{\Gamma}\left(R\left[x_{1}, \ldots, x_{n}\right]\right)$ is complete if and only if $\widetilde{\Gamma}\left(R\left[x_{1}\right]\right)$ is complete then the result follows from the theorem 1.10.

Remark 1.13. Let $R$ a ring such that $R$ is not isomorph to a subring of two integral domains, in particular if $R$ is a non-reduced ring, then $\widetilde{\Gamma}\left(R\left[x_{1}, \ldots, x_{n+1}\right]\right)$ is complete if and only if $Z^{\star}\left(\Gamma\left(R\left[x_{1}\right]\right)\right)$ is complete.

\section{Relation between $\Gamma(R), Z^{\star}(\Gamma(R))$ and $\widetilde{\Gamma}(R)$}

In this section, we will be interested in the cases where $\Gamma(R)$ or $Z^{\star}(\Gamma(R))$ coincides with $\widetilde{\Gamma}(R)$ and we provide sufficient and necessary conditions concerning these situations. The results are generalizations of our results concerning the finite case processed in [5].

As first step, we shall prove the following lemmas.

Lemma 2.1. $\widetilde{\Gamma}(R)=\Gamma(R)$ if and only if $\widetilde{\Gamma}(T(R))=\Gamma(T(R))$. 
Proof. $\Rightarrow)$ : Suppose that $\widetilde{\Gamma}(R)=\Gamma(R)$. Since $\Gamma(T(R))$ is a spanning subgraph of $\widetilde{\Gamma}(T(R))$, if two vertices of $\Gamma(T(R))$ are adjacent then they are adjacent as vertices of $\widetilde{\Gamma}(T(R))$. On the other hand, let $\frac{x}{s}, \frac{y}{t} \in Z(T(R))^{\star}$ such that $\frac{x}{s}$ and $\frac{y}{t}$ are adjacent in $\widetilde{\Gamma}(T(R))$. we suppose that $\frac{x}{s}+\frac{y}{t} \in Z(T(R))$, if not $\frac{x}{s} \frac{y}{t}=0$ hence $\frac{x}{s}$ and $\frac{y}{t}$ are adjacent in $\Gamma(T(R))$, so $t x+s y \in Z(R)$ hence $t x$ and $s y$ are adjacent in $\widetilde{\Gamma}(R)$ therefore $t x . s y=0$ because $\widetilde{\Gamma}(R)=\Gamma(R)$ so $x y=0$ and thus $\frac{x}{s} \frac{y}{t}=0$.

$\Leftarrow)$ : Since $\Gamma(R)$ is a spanning subgraph of $\widetilde{\Gamma}(R)$, if two vertices of $\Gamma(R)$ are adjacent then they are adjacent as vertices of $\widetilde{\Gamma}(R)$. Let $x, y \in Z(R)^{\star}$ such that $x$ and $y$ are adjacent in $\widetilde{\Gamma}(R)$. If $x . y=0$ then $x$ and $y$ are adjacent in $\Gamma(R)$; if not $x+y \in Z(R)$ so $\frac{x}{1}+\frac{y}{1} \in Z(T(R))^{\star}$ then $\frac{x}{1}$ and $\frac{y}{1}$ are adjacent in $\widetilde{\Gamma}(T(R))$ hence $\frac{x}{1} \frac{y}{1}=0$ because $\widetilde{\Gamma}(T(R))=\Gamma(T(R))$ and thus $x$ and $y$ are adjacent in $\Gamma(R)$.

Lemma 2.2. Let $R$ a decomposable ring. If $\widetilde{\Gamma}(R)=\Gamma(R)$, then $R \simeq \mathbb{Z}_{2}^{2}$.

Proof. Since $R$ is decomposable, there exists two non-trivial rings $R_{1}, R_{2}$ such that $R \simeq R_{1} \times R_{2}$. We claim that $R_{1} \simeq \mathbb{Z}_{2}$, indeed, suppose that there exists $x_{1} \in R_{1}$ such that $x_{1} \notin\{0,1\}$ so we consider $x=\left(x_{1}, 0\right), y=(1,0)$ then $x, y \in Z(R)^{\star}, x \neq y$ because $x_{1} \neq 1$. We have $x+y \in Z(R)$ and since $\widetilde{\Gamma}(R)=\Gamma(R), x y=0$ then $x_{1}=0$. In the same way, $R_{2} \simeq \mathbb{Z}_{2}$ and thus $R \simeq \mathbb{Z}_{2}^{2}$.

Theorem 2.3. $\widetilde{\Gamma}(R)=\Gamma(R)$ if and only if $\Gamma(R)$ is complete.

Proof. $\Leftarrow)$ : It is clear that if $\Gamma(R)$ is complete then $\widetilde{\Gamma}(R)=\Gamma(R)$.

$\Rightarrow)$ : We can suppose that $R$ is not isomorph to $\mathbb{Z}_{2}^{2}$, if not $\Gamma(R)$ is complete. We claim that $\forall x \in Z(R), x^{2}=0$, indeed, let $x \in Z(R)^{\star}$.

Case 1: If $2 x \neq 0$ so $x \neq-x$ then $x^{2}=0$ because $x$ and $-x$ are adjacent in $\widetilde{\Gamma}(R)$.

Case 2: If $2 x=0$, let $y \in Z(R)^{\star}$ such that $x y=0$.

We claim that $x+y \in Z(R)$, indeed, if $x+y \notin Z(R), e=\frac{x}{x+y} \in T(R)$ verify $e(1-e)=0$ in $T(R)$ so $e^{2}=e$ and since $e \notin\{0,1\}, T(R)$ is decomposable. On the other hand, since $\widetilde{\Gamma}(R)=\Gamma(R)$, according to lemma 2.1, $\widetilde{\Gamma}(T(R))=$ $\Gamma(T(R))$ hence, using lemma $2.2, T(R) \simeq \mathbb{Z}_{2}^{2}$ and thus $R \simeq \mathbb{Z}_{2}^{2}$.

If $x+y=0$, then $x^{2}=0$. If $x+y \neq 0, x+y \in Z(R)^{\star}, x \neq x+y$ and $x+(x+y)=y \in Z(R)$ so $x(x+y)=0$ then $x^{2}=0$.

We know that $N i l(R) \subset Z(R)$ and since $\forall x \in Z(R), x^{2}=0, N(R)=Z(R)$. If $x, y \in Z(R)^{\star}$ such that $x \neq y$ then $x+y \in Z(R)$ and thus $x . y=0$ because $\widetilde{\Gamma}(R)=\Gamma(R)$. 
According to theorem 2.8 [3] and the previous theorem, we obtain:

Corollary 2.4. $\widetilde{\Gamma}(R)=\Gamma(R)$ if and only either $R \simeq \mathbb{Z}_{2}^{2}$ or for all $x, y \in$ $Z(R), x y=0$.

Next, we turn to the situation where $\widetilde{\Gamma}(R)=Z^{\star}(\Gamma(R))$. Let's start by proving the following lemma.

Lemma 2.5. $\widetilde{\Gamma}(R)=Z^{\star}(\Gamma(R))$ if and only if $\widetilde{\Gamma}(T(R))=Z^{\star}(\Gamma(T(R)))$.

Proof. $\Rightarrow)$ : Suppose that $\widetilde{\Gamma}(T(R)) \neq Z^{\star}(\Gamma(T(R)))$ so there exists $\frac{x}{s}, \frac{y}{t} \in$ $Z(T(R))^{\star}$ such that $\frac{x}{s}$ and $\frac{y}{t}$ are adjacent in $\widetilde{\Gamma}(T(R))$ but not adjacent in $Z^{\star}(\Gamma(T(R)))$ hence $x y=0$ but $t x+s y \notin Z(R)$. We have $(t x),(s y) \in Z(R)^{\star}$, $(t x)(s y)=0$ and $t x \neq s y$ (because $\left.\frac{x}{s} \neq \frac{y}{t}\right)$ so $(t x),(s y)$ are adjacent in $\widetilde{\Gamma}(R)$. However, $(t x),(s y)$ are not adjacent in $Z^{\star}(\Gamma(R))$ and thus $\widetilde{\Gamma}(R) \neq Z^{\star}(\Gamma(R))$. $\Leftarrow)$ : Let $x, y \in Z(R)^{\star}$ such that $x$ and $y$ are adjacent in $\widetilde{\Gamma}(R)$. We suppose that $x . y=0$, if not $x+y \in Z(R)$ so $x$ and $y$ are adjacent in $Z^{\star}(\Gamma(R))$, then $\frac{x}{1} \frac{y}{1}=0$ thus $\frac{x}{1}, \frac{y}{1}$ are adjacent in $\widetilde{\Gamma}(T(R))$ then $\frac{x}{1}+\frac{y}{1} \in Z(T(R))$ because $\widetilde{\Gamma}(T(R))=Z^{\star}(\Gamma(T(R)))$ therefore $x+y \in Z(R)$.

Theorem 2.6. $\widetilde{\Gamma}(R)=Z^{\star}(\Gamma(R))$ if and only if $T(R)$ is indecomposable.

Proof. $\Rightarrow)$ : Suppose that $T(R)$ is decomposable so there exists a nontrivial idempotent $e \in T(R)$ then $e, 1-e \in Z(T(R))^{\star}$ because $e(1-e)=0$. Also, $e \neq 1-e$, if not $e=e^{2}=0$, so $e, 1-e$ are adjacent in $\widetilde{\Gamma}(T(R))$, however $e$ and $1-e$ are not adjacent in $Z^{\star}(\Gamma(T(R)))$ because $e+(1-e)=1 \notin$ $Z(T(R))$ then $\widetilde{\Gamma}(T(R)) \neq Z^{\star}(\Gamma(T(R)))$ therefore, according to the lemma $2.5, \widetilde{\Gamma}(R) \neq Z^{\star}(\Gamma(R))$.

$\Leftarrow)$ : Suppose that $\widetilde{\Gamma}(R) \neq Z^{\star}(\Gamma(R))$ so there exists $x, y \in Z(R)^{\star}$ such that $x, y$ are adjacent in $\widetilde{\Gamma}(R)$ but $x, y$ are not adjacent in $Z^{\star}(\Gamma(R))$ so $x y=0$ and $x+y \notin Z(R)$. We consider $e=\frac{x}{x+y} \in T(R)$, we have $e \notin\{0,1\}$ and $e(1-e)=\frac{x y}{(x+y)^{2}}=0$ so $e=e^{2}$ then there is a nontrivial idempotent in $T(R)$ thus $T(R)$ is decomposable.

\section{Examples 2.7.}

- In [5], we showed that if $R$ is a finite ring, $\widetilde{\Gamma}(R)=Z^{\star}(\Gamma(R))$ if and only if $Z^{\star}(\Gamma(R))$ is a complete graph (i.e., $Z(R)$ is an ideal of $R$ ). However, in general, this result is false: it is obvious that if $Z(R)$ is an ideal of $R$, then $\widetilde{\Gamma}(R)=Z^{\star}(\Gamma(R))$ but the converse is false as shown by the following example: we consider the commutative ring $R=\mathbb{Z}(+) \mathbb{Z}_{6}$ the idealization of the $\mathbb{Z}$-module $\mathbb{Z}_{6}$ in $\mathbb{Z}$ (cf. [8]). According to theorem 
25.3 [8], $(r, x) \in R$ is a zero-divisor of $R$ if and only if $r \in Z(\mathbb{Z}) \cup Z\left(\mathbb{Z}_{6}\right)$ so $(r, x) \in R$ is a zero-divisor of $R$ if and only if $r \in 2 \mathbb{Z} \cup 3 \mathbb{Z}$. We claim that $\widetilde{\Gamma}(R)=Z^{\star}(\Gamma(R))$, indeed, let $a=(n, x), b=(m, y) \in$ $Z(R)^{\star}$ such that $a b=0$ so $n m=0$ then $n=0$ or $m=0$ and thus $a+b \in Z(R)$. On the other hand, $Z(R)$ is not an ideal of $R$, indeed, $(-2,0),(3,0) \in Z(R)$ but $(-2,0)+(3,0)=(1,0) \notin Z(R)$. Also, we note that $\widetilde{\Gamma}(R)=Z^{\star}(\Gamma(R))$ but $\widetilde{\Gamma}(R)$ is not complete.

- It is obvious that, in general, if $T(R)$ is indecomposable, $R$ is not necessarily local, indeed, $\mathbb{Z}$ is not local but $T(\mathbb{Z})=\mathbb{Q}$ is indecomposable. For an other example with nontrivial graphs, just consider the same ring $R=\mathbb{Z}(+) \mathbb{Z}_{6}$ thus $T(R)$ is indecomposable and using theorem 25.1

(3) [8], $R$ is not local.

\section{Corollary 2.8.}

(a) Let $R$ such that every non-unit is a zero-divisor. Then, $\widetilde{\Gamma}(R)=Z^{\star}(\Gamma(R))$ if and only if $R$ is indecomposable.

(b) Let $R$ such that $\operatorname{dim} R=0$. Then, $\widetilde{\Gamma}(R)=Z^{\star}(\Gamma(R))$ if and only if $R$ is indecomposable.

(c) Let $R$ a noetherian reduced ring. $\widetilde{\Gamma}(R)=Z^{\star}(\Gamma(R))$ if and only if $Z^{\star}(\Gamma(R))$ is complete (i.e., $Z(R)$ is an ideal).

(d) Let $R$ an artinian ring. $\widetilde{\Gamma}(R)=Z^{\star}(\Gamma(R))$ if and only if $Z^{\star}(\Gamma(R))$ is complete (i.e., $Z(R)$ is an ideal).

Proof. (a) follows immediately from $R \simeq T(R)$.

(b) Let $x$ a non-unit element of $R$ so there exist a minimal prime ideal $\mathfrak{p}$ of $R$ such that $x \in \mathfrak{p}$ because $\operatorname{dim} R=0$. Then, according to the theorem 2.1 [8], there exist $y \notin \mathfrak{p}$ and an integer $n>0$ such that $y x^{n}=0$. Let $k>0$ the smallest integer such $y x^{k}=0$ so $\left(y x^{k-1}\right) x=0$ and since $y x^{k-1} \neq 0$ so $x \in Z(R)$.

(c) Since $R$ is a noetherian reduced ring, $\bigcap_{i=1}^{n} \mathfrak{p}_{i}=\{0\}$, where $\mathfrak{p}_{1}, \ldots, \mathfrak{p}_{n}$ are the minimal prime ideals of $R$. We claim that if $n \geq 2$, then $T(R)$ is decomposable, indeed, suppose that $n \geq 2$ then, according to the prime avoidance lemma, $\mathfrak{p}_{1} \not \subseteq \bigcup_{i=2}^{n} \mathfrak{p}_{i}$ and $\bigcap_{i=2}^{n} \mathfrak{p}_{i} \nsubseteq \nsubseteq \mathfrak{p}_{1}$. Let $x \in \mathfrak{p}_{1}$ such the $x \notin \bigcup_{i=2}^{n} \mathfrak{p}_{i}$ and $y \in \bigcap_{i=2}^{n}$ such that $y \notin \mathfrak{p}_{1}$ so $x+y \notin \bigcup_{i=1}^{n} \mathfrak{p}_{i}=Z(R)$ and since $x y \in \bigcap_{i=2}^{n} \mathfrak{p}_{i}=$ 0 then $e=\frac{x}{x+y}$ is a non-trivial idempotent in $T(R)$. 
(c) Suppose that $R$ is artinian then $\operatorname{dim} R=0$ so, by (b), $\widetilde{\Gamma}(R)=Z^{\star}(\Gamma(R))$ if and only if $R$ is indecomposable. Using Theorem 8.7 [1], $R \simeq \prod_{i=1}^{n} R_{i}$, where $R_{1}, \ldots, R_{n}$ are local rings and thus the result is an immediate consequence.

\section{Others Properties}

In this section, we give some other properties of $\widetilde{\Gamma}(R)$.

Theorem 3.1. If $\left|Z(R)^{\star}\right| \geq 3, \widetilde{\Gamma}(R)$ is hypertriangulated, i.e., every edge is in a triangle.

Proof. Let $x, y \in Z(R)^{\star}$ such that $x$ and $y$ are adjacent and $z \in Z(R)^{\star}$ such that $z \notin\{x, y\}$. If $x, y \in N(z)$, where $N(z)$ denotes the set of vertices that are adjacent to $z$, then $x y z$ is a triangle. Thus we can suppose that $x \notin N(z)$ (by symmetry the other case is similar). Let $a, b, c \in Z(R)^{\star}$ such that $a x=b y=c z=0$.

Case 1: If $x y=0$ : Then $c y(x+z)=0$ so $c y=0$ because $x \notin N(z)$, hence $y(x+c)=0$ therefore $x+c \in Z(R)$. On the other hand, $c \neq x$, if not $x z=c z=0 ; c \neq y$, if not $y(x+z)=0$ then $y=0$. Thus $x y c$ is a triangle.

Case 2: If $x y \neq 0$ : If $y z=0, a y(x+z)=0$ so $a y=0$ because $x \notin N(z)$. Also, $a \neq x$, if not $x y=a y=0 ; a \neq y$, if not $x y=x a=0$. Therefore $x a y$ is a triangle. If $y z \neq 0$. Since $a c(x+z)=0$ and $x \notin N(z), a c=0$. There are two case: If $b c=0, b(y+c)=0$ so $y+c \in Z(R)$. In this case, $c \neq x$, if not $x z=c z=0 ; c \neq y$, if not $y z=c z=0$. Therefore $x y c$ is a triangle. If $b c \neq 0, a(b c+x)=0$ and $(b c) y=0$. Also, $b c \neq x$, if not $x z=b c z=0$; $b c \neq y$, if not $y z=b c z=0$. Thus $x y(b c)$ is a triangle.

Remark 3.2. it is clear that the result (a) of theorem 3.3 [5] is an immediate consequence of the previous theorem.

We recall that an ideal is called decomposable if it admits a primary decomposition.

Theorem 3.3. If the zero ideal is decomposable, in particular if $R$ is a noetherian ring, then there exists $a \in Z(R)^{\star}$ such that for every $x \in Z(R)^{\star} \backslash$ $\{a\}$, $a$ and $x$ are adjacent.

Proof. Since $(0)$ is is decomposable, $Z(R)=\bigcup_{i=1}^{n} \mathfrak{p}_{i}$, where $\mathfrak{p}_{i}=\left(0: a_{i}\right)$ are the associated ideals to (0). If $n=1$, the result is trivial so we can 
suppose that $n \geq 2$. Also, if $a_{1} \ldots a_{n} \neq 0$, let $a=a_{1} \ldots a_{n}$ so $a \in Z(R)^{\star}$ and $\forall x \in Z(R)^{\star}$, there exists $i$ such that $x \in \mathfrak{p}_{i}=\left(0: a_{i}\right)$ so $a x=0$. Suppose that $a_{1} \ldots a_{n}=0$, let $X=\left\{m \in\{1, \ldots, n-1\} / \exists\left\{a_{i_{1}}, \ldots, a_{i_{m}}\right\} \subset\right.$ $\left.\left\{a_{1}, \ldots, a_{m}\right\}: a_{i_{1}} \ldots a_{i_{m}} \neq 0\right\}$. We have $X \neq \emptyset$ because $a_{1} \neq 0$. Let $k=\max (X)$ and $a=a_{i_{1}} \ldots a_{i_{k}} \neq 0$. Then, $\forall x \in Z(R)^{\star}$, there exists $i$ such that $x \in \mathfrak{p}_{i}=\left(0: a_{i}\right)$. If $i \in\left\{i_{1}, \ldots, a_{k}\right\}$ then $a x=0$. If $i \notin\left\{i_{1}, \ldots, a_{k}\right\}$ so $a a_{i}=0$ because $k=\max (X)$ and since $x a_{i}=0$ then $a_{i}(x+a)=0$ hence $x+a \in Z(R)$.

Theorem 3.4. If $R$ is a finite ring, then $\widetilde{\Gamma}(R)$ is hamiltonian.

Proof. Since $R$ is finite, then $R \simeq R_{1} \times \ldots R_{n}$, where $R_{1}, \ldots, R_{n}$ are local rings. We can suppose that $n \geq 2$, if not $\widetilde{\Gamma}(R)$ is complete. Up to isomorphism, $Z(R)=M_{1} \cup \ldots M_{n}$, where $M_{i}=R_{1} \times \ldots \times \mathfrak{m}_{i} \times \cdots \times R_{n}$ and $\mathfrak{m}_{i}$ is the maximal ideal of $R_{i}$. Let $X_{1}=M_{1} \backslash\{0\}$ and for every $i=2, \ldots, n$, $X_{i}=M_{i} \backslash\left(\bigcup_{j=1}^{i-1} M_{j}\right)$ then $Z(R)^{\star}=\bigcup_{i=1}^{n} X_{i}$ and $X_{i} \cap X_{j}=\emptyset$ if $i \neq j$.

Case where $n=2$ : If $\mathfrak{m}_{1}=\{0\}$ and $\mathfrak{m}_{2}=\{0\}$ then $R_{1}, R_{2}$ are fields and thus $\widetilde{\Gamma}(R)$ is complete (cf. theorem $2.4[5]$ ) so we can suppose that $\mathfrak{m}_{1} \neq\{0\}$ and consider $X_{1}$ as the orderly sequence $x_{11}, \ldots, x_{1 p_{1}}$, with $x_{11}=(0,1)$ and $x_{1 p_{1}}=(a, 0)$, where $a \in \mathfrak{m}_{1} \backslash\{0\}$. Also, we consider $X_{2}$ as the orderly sequence $x_{21}, x_{2 p_{2}}$ with $x_{2 p_{2}}=(1,0)$ so $x_{11}-\ldots-x_{1 p_{1}}$ (because $\left.x_{11}, \ldots, x_{1 p_{1}} \in M_{1}\right), x_{1 p_{1}}-x_{21}-\ldots-x_{2 p_{2}}$ (because they are in $M_{2}$ ) and $x_{2 p_{2}}-x_{11}$ (because $x_{2 p_{2}} x_{11}=0$ ) and thus we obtain the hamiltonian cycle $x_{11}-\ldots-x_{1 p_{1}}-x_{21}-\ldots-x_{2 p_{2}}-x_{11}$.

Case where $n \geq 3$ : If for every $i,\left|R_{i}\right|=2$, then $R$ id boolean and thus $\widetilde{\Gamma}(R)$ is complete (cf. theorem $2.4[5]$ ) so we can suppose that $\left|R_{n}\right| \geq 3$. We consider $X_{1}$ as the orderly sequence $x_{11}, \ldots, x_{1 p_{1}}$ with $x_{11}=(0, \ldots, 0,1)$, $x_{1 p_{1}}=(0, \ldots, 0, a)$ and $a \in R_{n} \backslash\{0,1\}$. Also, for every $i=2, \ldots, n$, we consider $X_{i}$ as the orderly sequence $x_{i 1}, \ldots, x_{i p_{i}}$ with $x_{i p_{i}}=(1, \ldots, 1,0, \ldots, 0)$ (1 being repeated (i-1) times) so for $i=1, \ldots, n-1, x_{i p_{i}} \in X_{i} \cap M_{i+1}$. Then, $x_{11}-\ldots-x_{1 p_{1}}$ (because they are in $M_{1}$ ) and for every $i=2, \ldots, n$, $x_{i-1 p_{i-1}}-x_{i 1}-\ldots-x_{i p_{i}}$ (because they are in the same $M_{i}$ ) and $x_{n p_{n}}-x_{11}$ (because $x_{n p_{n}} x_{11}=0$ ) therefore we obtain the hamiltonian cycle $x_{11}-\ldots$ $-x_{1 p_{1}}-x_{21}-\ldots-x_{2 p_{2}}-x_{31}-\ldots-x_{n p_{n}}-x_{11}$.

\section{References}

[1] M.F. Atiyah, I.G. Macdonald, Introduction to Commutative Algebra, Addison-Wesley Pub- lishing Company, 1969. 
[2] D. F. Anderson, A. Badawi, The total graph of a commutative ring. J. Algebra 320(7), 27062719 (2008)

[3] D. F. Anderson, P.S. Livingston, The zero-divisor graph of a commutative ring, J. Algebra 217 (1999), 434-447.

[4] I. Beck, Coloring of commutative rings, J. Algebra 116 (1988), 208-226.

[5] A. Cherrabi, H. Essannouni, E. Jabbouri, A. Ouadfel, On a new extension of the zero-divisor graph. Submit for publication

[6] R. Diestel, Graph Theory, Springer-Verlag, New York, 1997.

[7] M. Hazewinkel, N. Gubareni, V.V. Kirichenko, Algebras, Rings and Modules, Volume 1, Mathematics and its applications, Kluwer Academic Publishers, 2005.

[8] J. Huckaba, Commutative Rings with Zero Divisors, Dekker, New York, 1988.

[9] T.G. Lucas, The diameter of zero divisor graph, Journal of Algebra, 301 (2006), 174-193. 